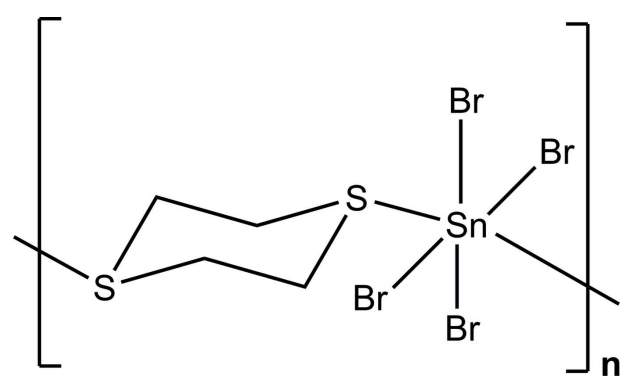

\title{
Crystal structure of a one-dimensional coordination polymer of tin(IV) bromide with 1,4-dithiane
}

\author{
Hans Reuter, ${ }^{*}$ Natalia Röwekamp-Krugley, Marius \\ Imwalle, Simona Keil and Martin Reichelt
}

Institute of Chemistry of New Materials, University of Osnabrueck, Barbarstr. 7, 49069 Osnabrueck, Germany. *Correspondence e-mail: hreuter@uos.de

Received 2 December 2015; accepted 12 December 2015

Edited by M. Nieger, University of Helsinki, Finland

The title compound, $\left[\mathrm{SnBr}_{4}\left(\mathrm{C}_{4} \mathrm{H}_{8} \mathrm{~S}_{2}\right)\right]$ \{systematic name: catenapoly[[tetrabromidotin(IV)]- $\mu-1,4-$ dithiane- $\left.\left.\kappa^{2} S: S^{\prime}\right]\right\}$, represents the first 1,4-dithiane complex with tin as coordination centre. The asymmetric unit consist of half a formula unit with the tin(IV) atom at the centre of symmetry at 0,0,1/2 (Wyckoff symbol $b$ ) and a centrosymmetric 1,4-dithiane molecule with the centre of symmetry in 1/2,0,1 (Wyckoff symbol $c$ ). The tin(IV) atom is coordinated in a distorted octahedral manner by the four bromine atoms and two sulfur atoms of two 1,4dithiane molecules in a trans-position. $\mathrm{Sn}-\mathrm{Br}$ [mean value: $2.561(5) \AA]$ and $\mathrm{Sn}-\mathrm{S}$ distances [2.6546 (6) $\AA]$ are in the typical range for octahedrally coordinated tin(IV) atoms and the dithiane molecule adopts a chair conformation. The onedimensional polymeric chains propagate along the [101] direction with weak intermolecular $\mathrm{Br} \cdots \mathrm{Br}$ [3.5724 (4) Å] between parallel chains and weak $\mathrm{Br} \cdot \mathrm{H}$ interactions [2.944$2.993 \AA$ ] within the chains.

Keywords: crystal structure; tin(IV) bromide; 1,4-dithiane; coordination polymer; $\mathrm{Br}$... Br interactions; $\mathrm{Br}$...H interactions.

CCDC reference: 1442283

\section{Related literature}

For the structural parameters in macrocyclic thioether complexes with $\mathrm{SnBr}_{4}$, see: Levason et al. (2003), and for dithioether complexes with $\mathrm{SnBr}_{4}$, see: Dann et al. (1996). For the oxidation of tin(II) to tin(IV), see: Deacon et al. (1997).

\section{Experimental}

$$
\begin{aligned}
& \text { 2.1. Crystal data } \\
& {\left[\mathrm{SnBr}_{4}\left(\mathrm{C}_{4} \mathrm{H}_{8} \mathrm{~S}_{2}\right)\right]} \\
& M_{r}=558.55 \\
& \text { Monoclinic, } P 2_{b} / n \\
& a=7.1033(4) \AA \\
& b=12.0526(8) \AA \\
& c=7.4032(5) \AA \\
& \beta=112.144(2)^{\circ}
\end{aligned}
$$

$$
\begin{aligned}
& V=587.06(7) \AA^{3} \\
& Z=2 \\
& \text { Mo } K \alpha \text { radiation } \\
& \mu=16.09 \mathrm{~mm}^{-1} \\
& T=100 \mathrm{~K} \\
& 0.16 \times 0.06 \times 0.06 \mathrm{~mm}
\end{aligned}
$$

\subsection{Data collection}

\section{Bruker APEXII CCD} diffractometer

Absorption correction: multi-scan (SADABS; Bruker, 2009) $T_{\min }=0.182, T_{\max }=0.450$

\subsection{Refinement}

$R\left[F^{2}>2 \sigma\left(F^{2}\right)\right]=0.017$

$w R\left(F^{2}\right)=0.036$

$S=1.14$

1426 reflections

54 parameters

$\mathrm{H}$-atom parameters constrained

$\Delta \rho_{\max }=0.72 \mathrm{e} \AA^{-3}$

$\Delta \rho_{\min }=-0.46$ e $\AA^{-3}$

Table 1

Selected contacts $(\AA)$.

\begin{tabular}{llll}
\hline $\mathrm{Br} 1 \cdots \mathrm{H} 11^{\mathrm{i}}$ & 2.965 & $\mathrm{Br} 1 \cdots \mathrm{H} 12^{\mathrm{iv}}$ & 3.078 \\
$\mathrm{Br} 1 \cdots \mathrm{H} 21^{\mathrm{ii}}$ & 2.993 & $\mathrm{Br} 1 \cdots \mathrm{H} 11^{\mathrm{v}}$ & 3.079 \\
$\mathrm{Br} 2 \cdots \mathrm{H} 22^{\mathrm{iii}}$ & 2.944 & $\mathrm{Br} 1 \cdots \mathrm{Br} 2^{\mathrm{vi}}$ & $3.5724(4)$ \\
\hline
\end{tabular}

Symmetry codes: (i) $-x,-y,-z+1$; (ii) $-x+1,-y,-z+2$; (iii) $x-1, y, z-1$; (iv) $x, y, z-1 ;(\mathrm{v})-x+\frac{1}{2}, y-\frac{1}{2},-z+\frac{3}{2}$; (vi) $-x+\frac{1}{2}, y-\frac{1}{2},-z+\frac{1}{2}$.

Data collection: APEX2 (Bruker, 2009); cell refinement: SAINT (Bruker, 2009); data reduction: $S A I N T$; $\operatorname{program}(\mathrm{s})$ used to solve structure: SHELXS97 (Sheldrick, 2008); program(s) used to refine structure: SHELXL2014/7 (Sheldrick, 2015); molecular graphics: DIAMOND (Brandenburg, 2006) and Mercury (Macrae et al., 2008); software used to prepare material for publication: SHELXTL (Sheldrick, 2008).

\section{Acknowledgements}

We thanks the state of Lower-Saxony and the Deutsche Forschungsgemeinschaft for funding the diffractometer.

Supporting information for this paper is available from the IUCr electronic archives (Reference: NR2064). 


\section{data reports}

\section{References}

Brandenburg, K. (2006). DIAMOND. Crystal Impact GbR, Bonn, Germany. Bruker (2009). APEX2, SADABS and SAINT. Bruker AXS Inc., Madison, Wisconsin, USA.

Dann, S. E., Genge, A. R. J., Levason, W. \& Reid, G. (1996). J. Chem. Soc. Dalton Trans. pp. 4471-4478.

Deacon, P. R., Mahon, M. F., Molloy, K. C. \& Waterfield, P. C. (1997). J. Chem. Soc. Dalton Trans. pp. 3705-3712.
Levason, W., Matthews, M. L., Patel, R., Reid, G. \& Webster, M. (2003). New J. Chem. 27, 1784-1788.

Macrae, C. F., Bruno, I. J., Chisholm, J. A., Edgington, P. R., McCabe, P., Pidcock, E., Rodriguez-Monge, L., Taylor, R., van de Streek, J. \& Wood, P. A. (2008). J. Appl. Cryst. 41, 466-470.

Sheldrick, G. M. (2008). Acta Cryst. A64, 112-122.

Sheldrick, G. M. (2015). Acta Cryst. C71, 3-8. 


\section{supporting information}

Acta Cryst. (2015). E71, m267-m268 [https://doi.org/10.1107/S2056989015023932]

\section{Crystal structure of a one-dimensional coordination polymer of tin(IV) bromide} with 1,4-dithiane

\section{Hans Reuter, Natalia Röwekamp-Krugley, Marius Imwalle, Simona Keil and Martin Reichelt}

S1. Synthesis and crystallization

A mixture of $0.55 \mathrm{~g}(2 \mathrm{mmol}) \mathrm{SnBr}_{2}$ and $0.24 \mathrm{~g}(2 \mathrm{mmol})$ 1,4-dithiane was heated in a closed ampule to $130{ }^{\circ} \mathrm{C}$ for 6 hours. No special care was taken to exclude oxygen or humidity. After cooling, the ampule was opened and its solid content inspected by optical microscopy. Only one fragment, a yellow needle-like crystal of the title compound proved to be suitable for single crystal X-ray diffraction. The presence of tin(IV) in the title compound instead of tin(II) demonstrates the complexity of reactions that must have taken place. Sensitivity of tin(II) compounds towards oxidation by air, however, is not unusual and well documented in literature (e.g. Deacon et al, 1997).

\section{S1.1. Refinement}

All hydrogen atoms could be localized in difference Fourier syntheses but were refined in geometric positions riding on the carbon atoms with $\mathrm{C}-\mathrm{H}$ distances of $0.99 \AA\left(-\mathrm{CH}_{2}-\right)$ and one common, free refined isotropic displacement factor.

\section{S2. Results and discussion}

Only some few coordination compounds of tin(IV) bromide with Lewis-bases containing two or more S-atoms as Lewisbase centers have been structurally characterized. The main structural features are one-dimensional chain structures in case of macrocyclic thioether complexes (Levason et al., 2003) with the Lewis-base molecules in a cis-, and transposition, respectiveley, and the formation of monomeric complexes as a result of chelatization in case of open chain dithioether molecules (Dann et al., 1996). In all cases, the tin atoms are octahedrally coordinated with similar $\mathrm{Sn}-\mathrm{Br}$ and $\mathrm{Sn}-\mathrm{S}$ bond lengths. 


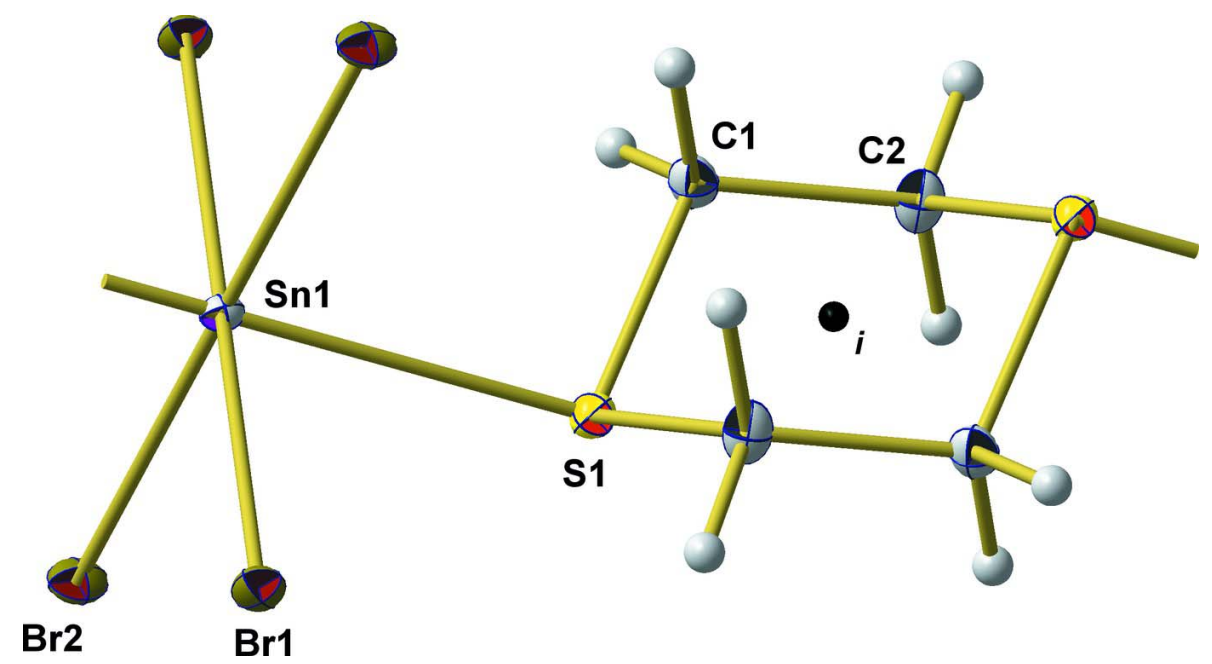

Figure 1

Ball-and-stick model of the asymmetric unit of the title compound with the atomic numbering scheme used. For a better understanding the asymmetric unit of the 1,4-dithiane molecule has been extended by its symmetry-related atoms generated by the centre of symmetry $i$ (black dot) at 1/2,0,1. With exception of the $\mathrm{H}$ atoms, which are shown as spheres of arbitrary radius, all atoms are drawn as displacement ellipsoids at the $50 \%$ probability level.

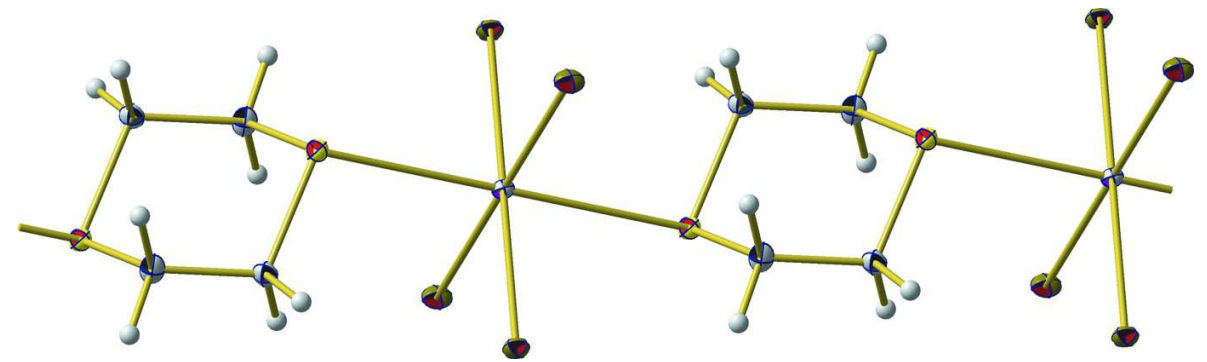

Figure 2

Part of the one-dimensional coordination polymer showing two complete building units. 


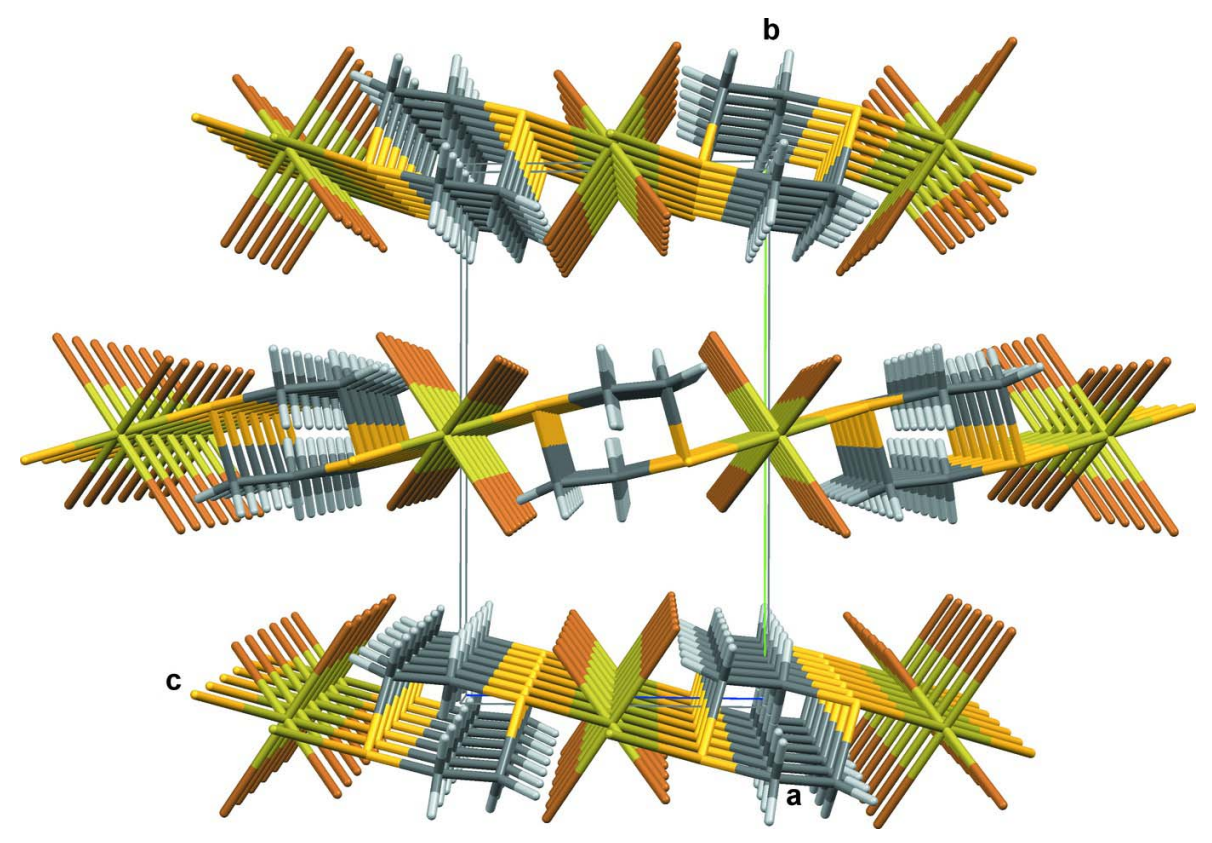

Figure 3

Perspective view of the crystal structure looking down the $a$ axis.

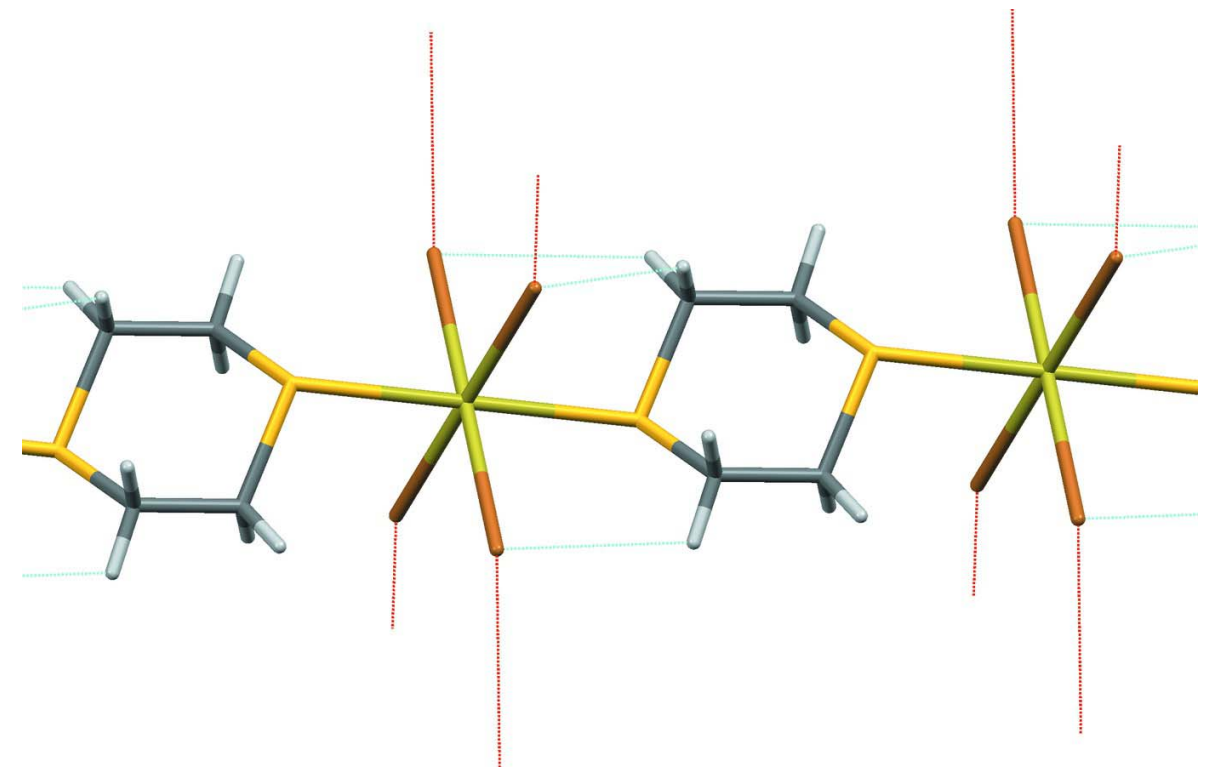

Figure 4

Shortest intrachain $\mathrm{H}^{\cdots} \cdots \mathrm{Br}$ (blue) and interchain $\mathrm{Br} \cdots \mathrm{Br}$ (red) interactions. 


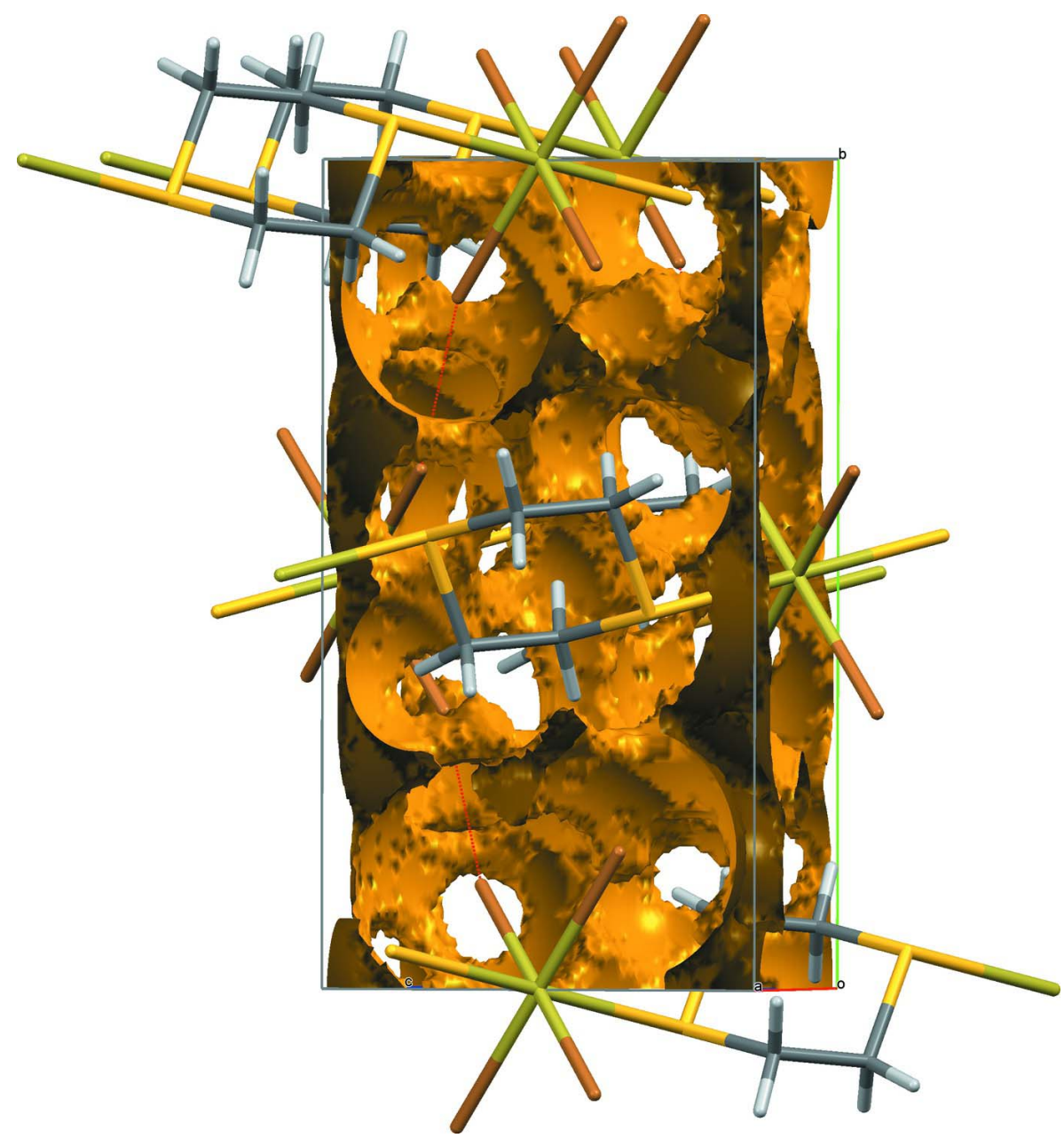

Figure 5

Three-dimensional representation of the contact surface (probe radius $=0.2 \AA$, outside color $=$ yellow, inside color $=$ brown) within the unit cell visualizing $\mathrm{Br} \cdots \mathrm{Br}$ interactions (red) between neighboring chains through holes in the surface.

catena-Poly[[tetrabromidotin(IV)]- $\mu-1,4-$ dithiane- $\left.\kappa^{2} S: S^{\prime}\right]$

Crystal data

$\left[\mathrm{SnBr}_{4}\left(\mathrm{C}_{4} \mathrm{H}_{8} \mathrm{~S}_{2}\right)\right]$

$M_{r}=558.55$

Monoclinic, $P 2{ }_{1} / n$

$a=7.1033(4) \AA$

$b=12.0526(8) \AA$

$c=7.4032(5) \AA$

$\beta=112.144(2)^{\circ}$

$V=587.06(7) \AA^{3}$

$Z=2$

\section{Data collection}

\section{Bruker APEXII CCD}

diffractometer

$\varphi$ and $\omega$ scans
$F(000)=508$

$D_{\mathrm{x}}=3.160 \mathrm{Mg} \mathrm{m}^{-3}$

Mo $K \alpha$ radiation, $\lambda=0.71073 \AA$

Cell parameters from 9935 reflections

$\theta=3.4-28.7^{\circ}$

$\mu=16.09 \mathrm{~mm}^{-1}$

$T=100 \mathrm{~K}$

Needle, yellow

$0.16 \times 0.06 \times 0.06 \mathrm{~mm}$

Absorption correction: multi-scan

(SADABS; Bruker, 2009)

$T_{\min }=0.182, T_{\max }=0.450$

22217 measured reflections 
1426 independent reflections

1339 reflections with $I>2 \sigma(I)$

$R_{\text {int }}=0.066$

$\theta_{\max }=28.0^{\circ}, \theta_{\min }=3.4^{\circ}$

\section{Refinement}

Refinement on $F^{2}$

Least-squares matrix: full

$R\left[F^{2}>2 \sigma\left(F^{2}\right)\right]=0.017$

$w R\left(F^{2}\right)=0.036$

$S=1.14$

1426 reflections

54 parameters

0 restraints

Primary atom site location: structure-invariant direct methods

Secondary atom site location: difference Fourier map

$$
\begin{aligned}
& h=-9 \rightarrow 9 \\
& k=-15 \rightarrow 15 \\
& l=-9 \rightarrow 9
\end{aligned}
$$

Hydrogen site location: inferred from neighbouring sites

$\mathrm{H}$-atom parameters constrained

$w=1 /\left[\sigma^{2}\left(F_{\mathrm{o}}^{2}\right)+(0.0043 P)^{2}+0.5753 P\right]$

where $P=\left(F_{\mathrm{o}}{ }^{2}+2 F_{\mathrm{c}}{ }^{2}\right) / 3$

$(\Delta / \sigma)_{\max }<0.001$

$\Delta \rho_{\max }=0.72 \mathrm{e} \AA^{-3}$

$\Delta \rho_{\min }=-0.46$ e $\AA^{-3}$

Extinction correction: SHELXL2014/7

(Sheldrick 2015),

$\mathrm{Fc}^{*}=\mathrm{kFc}\left[1+0.001 \mathrm{xFc}^{2} \lambda^{3} / \sin (2 \theta)\right]^{-1 / 4}$

Extinction coefficient: 0.0042 (3)

\section{Special details}

Geometry. All esds (except the esd in the dihedral angle between two 1.s. planes) are estimated using the full covariance matrix. The cell esds are taken into account individually in the estimation of esds in distances, angles and torsion angles; correlations between esds in cell parameters are only used when they are defined by crystal symmetry. An approximate (isotropic) treatment of cell esds is used for estimating esds involving 1.s. planes.

Fractional atomic coordinates and isotropic or equivalent isotropic displacement parameters $\left(\AA^{2}\right)$

\begin{tabular}{lllll}
\hline & $x$ & $y$ & $z$ & $U_{\text {iso }} * U_{\text {eq }}$ \\
\hline Sn1 & 0.0000 & 0.0000 & 0.5000 & $0.00740(7)$ \\
Br1 & $0.17963(4)$ & $-0.12497(2)$ & $0.33464(3)$ & $0.01109(8)$ \\
Br2 & $0.04504(4)$ & $0.16589(2)$ & $0.30464(4)$ & $0.01267(8)$ \\
S1 & $0.36282(9)$ & $0.04681(5)$ & $0.76832(9)$ & $0.01033(13)$ \\
C1 & $0.3132(4)$ & $0.0756(2)$ & $0.9867(3)$ & $0.0119(5)$ \\
H11 & 0.2349 & 0.1455 & 0.9686 & $0.015(4)^{*}$ \\
H12 & 0.2295 & 0.0151 & 1.0078 & $0.015(4)^{*}$ \\
C2 & $0.5088(4)$ & $0.0860(2)$ & $1.1657(4)$ & $0.0129(5)$ \\
H21 & 0.4771 & 0.1146 & 1.2764 & $0.015(4)^{*}$ \\
H22 & 0.6002 & 0.1399 & 1.1388 & $0.015(4)^{*}$ \\
\hline
\end{tabular}

Atomic displacement parameters $\left(\AA^{2}\right)$

\begin{tabular}{lllllll}
\hline & $U^{11}$ & $U^{22}$ & $U^{33}$ & $U^{12}$ & $U^{13}$ & $U^{23}$ \\
\hline Sn1 & $0.00749(13)$ & $0.00663(12)$ & $0.00903(13)$ & $0.00013(8)$ & $0.00420(9)$ & $0.00008(8)$ \\
Br1 & $0.01171(14)$ & $0.01121(13)$ & $0.01229(14)$ & $0.00266(9)$ & $0.00672(10)$ & $-0.00103(9)$ \\
Br2 & $0.01522(15)$ & $0.00948(13)$ & $0.01585(14)$ & $0.00006(9)$ & $0.00873(11)$ & $0.00347(9)$ \\
S1 & $0.0096(3)$ & $0.0106(3)$ & $0.0110(3)$ & $0.0001(2)$ & $0.0041(2)$ & $0.0002(2)$ \\
C1 & $0.0122(12)$ & $0.0131(12)$ & $0.0102(12)$ & $0.0027(10)$ & $0.0041(10)$ & $-0.0012(10)$ \\
C2 & $0.0121(12)$ & $0.0117(12)$ & $0.0124(12)$ & $0.0029(10)$ & $0.0017(10)$ & $-0.0038(10)$ \\
\hline
\end{tabular}


Geometric parameters $\left(\AA,{ }^{o}\right)$

\begin{tabular}{|c|c|c|c|}
\hline $\mathrm{Sn} 1-\mathrm{Br} 2^{\mathrm{i}}$ & $2.5574(3)$ & $\mathrm{Br} 1-\mathrm{H} 11^{\mathrm{v}}$ & 3.0788 \\
\hline $\mathrm{Sn} 1-\mathrm{Br} 2$ & $2.5574(3)$ & $\mathrm{Br} 1-\mathrm{Br} 2^{\mathrm{vi}}$ & $3.5724(4)$ \\
\hline $\mathrm{Sn} 1-\mathrm{Br} 1$ & $2.5638(2)$ & $\mathrm{S} 1-\mathrm{C} 1$ & $1.813(2)$ \\
\hline $\mathrm{Sn} 1-\mathrm{Br} 1^{\mathrm{i}}$ & $2.5638(3)$ & $\mathrm{S} 1-\mathrm{C} 2^{\mathrm{ii}}$ & $1.816(3)$ \\
\hline $\mathrm{Sn} 1-\mathrm{S} 1^{\mathrm{i}}$ & $2.6546(6)$ & $\mathrm{C} 1-\mathrm{C} 2$ & $1.521(3)$ \\
\hline $\mathrm{Sn} 1-\mathrm{S} 1$ & $2.6546(6)$ & $\mathrm{C} 1-\mathrm{H} 11$ & 0.9900 \\
\hline $\mathrm{Br} 1-\mathrm{H} 11^{\mathrm{i}}$ & 2.9646 & $\mathrm{C} 1-\mathrm{H} 12$ & 0.9900 \\
\hline $\mathrm{Br} 1-\mathrm{H} 21^{\mathrm{ii}}$ & 2.9932 & $\mathrm{C} 2-\mathrm{S} 1^{\mathrm{ii}}$ & $1.816(3)$ \\
\hline $\mathrm{Br} 2-\mathrm{H} 22^{\mathrm{iii}}$ & 2.9438 & $\mathrm{C} 2-\mathrm{H} 21$ & 0.9900 \\
\hline $\mathrm{Br} 1-\mathrm{H} 12^{\mathrm{iv}}$ & 3.0783 & $\mathrm{C} 2-\mathrm{H} 22$ & 0.9900 \\
\hline $\mathrm{Br} 2-\mathrm{Sn} 1-\mathrm{Br} 2$ & 180.0 & $\mathrm{H} 21^{\mathrm{ii}}-\mathrm{Br} 1-\mathrm{H} 11^{\mathrm{v}}$ & 68.8 \\
\hline $\mathrm{Br} 2-\mathrm{Sn} 1-\mathrm{Br} 1$ & $90.092(9)$ & $\mathrm{H} 12^{\mathrm{iv}}-\mathrm{Br} 1-\mathrm{H} 11^{\mathrm{v}}$ & 143.5 \\
\hline $\mathrm{Br} 2-\mathrm{Sn} 1-\mathrm{Br} 1$ & $89.908(9)$ & $\mathrm{Sn} 1-\mathrm{Br} 1-\mathrm{Br} 2^{\mathrm{vi}}$ & $167.821(10)$ \\
\hline $\mathrm{Br} 2^{\mathrm{i}}-\mathrm{Sn} 1-\mathrm{Br} 1^{\mathrm{i}}$ & $89.908(9)$ & $\mathrm{H} 11^{\mathrm{i}}-\mathrm{Br} 1-\mathrm{Br} 2^{\mathrm{vi}}$ & 102.6 \\
\hline $\mathrm{Br} 2-\mathrm{Sn} 1-\mathrm{Br} 1^{\mathrm{i}}$ & $90.092(9)$ & $\mathrm{H} 21^{\mathrm{ii}}-\mathrm{Br} 1-\mathrm{Br} 2^{\mathrm{vi}}$ & 88.3 \\
\hline $\mathrm{Br} 1-\mathrm{Sn} 1-\mathrm{Br} 1^{\mathrm{i}}$ & 180.0 & $\mathrm{H} 12^{\mathrm{iv}}-\mathrm{Br} 1-\mathrm{Br} 2^{\mathrm{vi}}$ & 85.5 \\
\hline $\mathrm{Br} 2^{\mathrm{i}}-\mathrm{Sn} 1-\mathrm{S} 1^{\mathrm{i}}$ & $87.931(15)$ & $\mathrm{H} 11^{\mathrm{v}}-\mathrm{Br} 1-\mathrm{Br} 2^{\mathrm{vi}}$ & 58.0 \\
\hline $\mathrm{Br} 2-\mathrm{Sn} 1-\mathrm{S} 1^{\mathrm{i}}$ & $92.069(15)$ & $\mathrm{Sn} 1-\mathrm{Br} 2-\mathrm{H} 22^{\mathrm{iii}}$ & 79.0 \\
\hline $\mathrm{Br} 1-\mathrm{Sn} 1-\mathrm{S} 1^{\mathrm{i}}$ & $92.027(15)$ & $\mathrm{C} 1-\mathrm{S} 1-\mathrm{C} 2^{\mathrm{ii}}$ & $100.11(12)$ \\
\hline $\mathrm{Br} 1^{\mathrm{i}}-\mathrm{Sn} 1-\mathrm{S} 1^{\mathrm{i}}$ & $87.973(15)$ & $\mathrm{C} 1-\mathrm{S} 1-\mathrm{Sn} 1$ & $104.35(8)$ \\
\hline $\mathrm{Br} 2^{\mathrm{i}}-\mathrm{Sn} 1-\mathrm{S} 1$ & $92.069(15)$ & $\mathrm{C} 22^{\mathrm{ii}}-\mathrm{S} 1-\mathrm{Sn} 1$ & $105.15(8)$ \\
\hline $\mathrm{Br} 2-\mathrm{Sn} 1-\mathrm{S} 1$ & $87.931(15)$ & $\mathrm{C} 2-\mathrm{C} 1-\mathrm{S} 1$ & $111.83(17)$ \\
\hline $\mathrm{Br} 1-\mathrm{Sn} 1-\mathrm{S} 1$ & $87.973(15)$ & $\mathrm{C} 2-\mathrm{C} 1-\mathrm{H} 11$ & 109.2 \\
\hline $\mathrm{Br} 1{ }^{\mathrm{i}}-\mathrm{S} n 1-\mathrm{S} 1$ & $92.027(15)$ & $\mathrm{S} 1-\mathrm{C} 1-\mathrm{H} 11$ & 109.2 \\
\hline $\mathrm{S} 1{ }^{\mathrm{i}}-\mathrm{Sn} 1-\mathrm{S} 1$ & $180.00(3)$ & $\mathrm{C} 2-\mathrm{C} 1-\mathrm{H} 12$ & 109.2 \\
\hline $\mathrm{Sn} 1-\mathrm{Br} 1-\mathrm{H} 11^{\mathrm{i}}$ & 83.1 & $\mathrm{~S} 1-\mathrm{C} 1-\mathrm{H} 12$ & 109.2 \\
\hline $\mathrm{Sn} 1-\mathrm{Br} 1-\mathrm{H} 21^{\mathrm{ii}}$ & 83.6 & $\mathrm{H} 11-\mathrm{C} 1-\mathrm{H} 12$ & 107.9 \\
\hline $\mathrm{H} 11^{\mathrm{i}}-\mathrm{Br} 1-\mathrm{H} 21^{\mathrm{ii}}$ & 161.5 & $\mathrm{C} 1-\mathrm{C} 2-\mathrm{S} 1^{\mathrm{ii}}$ & $111.25(17)$ \\
\hline $\mathrm{Sn} 1-\mathrm{Br} 1-\mathrm{H} 12^{\mathrm{iv}}$ & 106.2 & $\mathrm{C} 1-\mathrm{C} 2-\mathrm{H} 21$ & 109.4 \\
\hline $\mathrm{H} 11^{\mathrm{i}}-\mathrm{Br} 1-\mathrm{H} 12^{\mathrm{iv}}$ & 80.0 & $\mathrm{~S} 1{ }^{\mathrm{ii}}-\mathrm{C} 2-\mathrm{H} 21$ & 109.4 \\
\hline $\mathrm{H} 21^{\mathrm{ii}}-\mathrm{Br} 1-\mathrm{H} 12^{\mathrm{iv}}$ & 116.1 & $\mathrm{C} 1-\mathrm{C} 2-\mathrm{H} 22$ & 109.4 \\
\hline $\mathrm{Sn} 1-\mathrm{Br} 1-\mathrm{H} 11^{\mathrm{v}}$ & 110.3 & $\mathrm{~S} 11^{\mathrm{ii}}-\mathrm{C} 2-\mathrm{H} 22$ & 109.4 \\
\hline $\mathrm{H} 11^{\mathrm{i}}-\mathrm{Br} 1-\mathrm{H} 11^{\mathrm{v}}$ & 104.2 & $\mathrm{H} 21-\mathrm{C} 2-\mathrm{H} 22$ & 108.0 \\
\hline $\mathrm{Br} 2-\mathrm{Sn} 1-\mathrm{S} 1-\mathrm{C} 1$ & $-60.87(9)$ & $\mathrm{Br} 1-\mathrm{Sn} 1-\mathrm{S} 1-\mathrm{C} 2^{\mathrm{ii}}$ & $-46.00(9)$ \\
\hline $\mathrm{Br} 2-\mathrm{Sn} 1-\mathrm{S} 1-\mathrm{C} 1$ & $119.13(9)$ & $\mathrm{Br} 1^{\mathrm{i}}-\mathrm{Sn} 1-\mathrm{S} 1-\mathrm{C} 2^{\mathrm{ii}}$ & $134.00(9)$ \\
\hline $\mathrm{Br} 1-\mathrm{Sn} 1-\mathrm{S} 1-\mathrm{C} 1$ & $-150.89(9)$ & $\mathrm{Sn} 1-\mathrm{S} 1-\mathrm{C} 1-\mathrm{C} 2$ & $170.73(16)$ \\
\hline 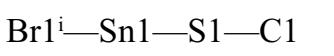 & $29.11(9)$ & $\mathrm{S} 1-\mathrm{C} 1-\mathrm{C} 2-\mathrm{S} 1^{\mathrm{ii}}$ & $-69.0(2)$ \\
\hline $\mathrm{Br} 2^{\mathrm{i}}-\mathrm{Sn} 1-\mathrm{S} 1-\mathrm{C} 2^{\mathrm{ii}}$ & $44.02(9)$ & $\mathrm{C} 1-\mathrm{C} 2-\mathrm{S} 1^{\mathrm{ii}}-\mathrm{C} 1^{\mathrm{ii}}$ & $61.7(2)$ \\
\hline $\mathrm{Br} 2-\mathrm{Sn} 1-\mathrm{S} 1-\mathrm{C}^{2 \mathrm{ii}}$ & $-135.98(9)$ & $\mathrm{C} 22^{\mathrm{ii}}-\mathrm{S} 1-\mathrm{C} 1-\mathrm{C} 2$ & $62.1(2)$ \\
\hline
\end{tabular}

Symmetry codes: (i) $-x,-y,-z+1$; (ii) $-x+1,-y,-z+2$; (iii) $x-1, y, z-1$; (iv) $x, y, z-1$; (v) $-x+1 / 2, y-1 / 2,-z+3 / 2$; (vi) $-x+1 / 2, y-1 / 2,-z+1 / 2$. 